\title{
Use of ultrafiltration during and after cardiopulmonary bypass in children
}

\author{
J. William Gaynor, MD
}

See related article on page 220 .

From the Division of Pediatric Cardiothoracic Surgery, The Children's Hospital of Philadelphia, Philadelphia, Pa.

Received for publication March 12, 2001; accepted for publication March 20, 2001.

Address for reprints: J. William Gaynor, MD, Division of Pediatric Cardiothoracic Surgery, The Children's Hospital of Philadelphia, 34th St and Civic Center Blvd, Philadelphia PA 19104 (E-mail: gaynor@email.chop.edu).

J Thorac Cardiovasc Surg 2001;122:209-11

Copyright (C) 2001 by The American Association for Thoracic Surgery

0022-5223/2001 \$35.00 + $0 \quad \mathbf{1 2 / 1 / 1 1 5 9 2 5}$

doi:10.1067/mtc.2001.115925

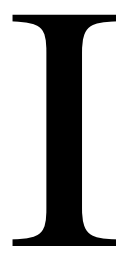

mprovements in the technology of cardiopulmonary bypass (CPB) have significantly reduced morbidity after repair of complex congenital heart defects, even in very small neonates. Use of CPB, however, may expose infants to extremes of hemodilution and hypothermia, often in association with tissue ischemia, as well as initiate a systemic inflammatory response with significant accumulation of excess body water. Organ dysfunction after $\mathrm{CPB}$, especially the heart, lungs, and brain, may result in significant postoperative morbidity and mortality. A variety of techniques have been developed to reverse the increase in total body water (TBW) after CPB, including ultrafiltration during $\mathrm{CPB}$, postoperative peritoneal dialysis, postoperative continuous arteriovenous hemofiltration, and aggressive use of diuretics postoperatively. Ultrafiltration is a technique that removes plasma water and low molecular weight solutes by a convective process using hydrostatic forces across a semipermeable membrane. The composition of the ultrafiltrate is dependent on the pore size of the hemofilter. Ultrafiltration was initially used during $\mathrm{CPB}$, usually during rewarming (conventional ultrafiltration or CUF). The volume of filtrate that can be removed during CUF is restricted by the volume of the venous reservoir, and thus CUF provides only a limited ability to remove excess water and reverse hemodilution.

Because of dissatisfaction with the ability of CUF to consistently prevent the increase in TBW and reverse hemodilution after CPB in infants, Naik, Knight and Elliott ${ }^{1,2}$ introduced a technique of ultrafiltration after separation from $\mathrm{CPB}$, which they termed modified ultrafiltration (MUF). In a preliminary study, they compared the efficacy of no ultrafiltration, CUF, and MUF in preventing accumulation of excess TBW. ${ }^{1}$ Changes in TBW were monitored by bioelectric impedance. The volume of filtrate that could be removed during MUF was significantly greater than during CUF. MUF significantly reduced the postoperative increase in TBW, whereas results with CUF were no different from control. CUF did prevent the increase in TBW in some patients; however, the response was neither uniform nor reproducible. In a prospective randomized trial, MUF was shown to significantly reduce the accumulation of TBW, decrease postoperative blood loss, and decrease postoperative blood product use when compared with no ultrafiltration. ${ }^{2}$ Unexpectedly, MUF resulted in a significant increase in arterial blood pressure. In a subsequent study, MUF was shown to increase cardiac index and decrease pulmonary vascular resistance with unchanged systemic vascular resistance. The hemodynamic benefits of MUF correlated directly with increasing hematocrit value and thus the degree of hemoconcentration. ${ }^{3}$

Since publication of these initial studies, there has been increasing interest in the use of ultrafiltration both during and after CPB. A MEDLINE keyword search identified 40 articles discussing MUF since 1995. Use of MUF has been shown to decrease TBW accumulation, postoperative blood loss, and blood product use; improve left ventricular systolic function, improve alveolar-arterial oxygen gradient and lung compliance; decrease the frequency of pulmonary hypertensive episodes; decrease the duration of postoperative ventilation; and decrease the incidence of pleural effusions after cavopulmonary connection and the Fontan procedure. ${ }^{1-7}$ Despite these reports of beneficial efforts, there has been increasing confusion and 
controversy concerning use of MUF. Much of this controversy stems from the lack of a standard definition or method for MUF. In the initial report, MUF was performed in an arteriovenous fashion. ${ }^{1,2}$ After separation from CPB, blood is withdrawn from the aortic cannula and is passed through the hemofilter, and the concentrated blood is returned to the right atrium. As fluid is removed from the patient, the filling pressures decrease. Blood from the bypass circuit is concentrated with the filter and infused to maintain intravascular volume. MUF thus provides the capability to remove excess water from the patient and salvage blood from the bypass circuit. MUF is continued until the hematocrit value is $40 \%$ or no blood remains in the bypass circuit. Some studies have used venovenous MUF, in which blood is withdrawn from the right atrium and returned to the right atrium. No direct comparisons of the effectiveness of arteriovenous MUF and venovenous MUF have been performed. Journois and colleagues ${ }^{8}$ reported a modification of CUF, which they termed zero-balance ultrafiltration, in which ultrafiltration is performed during rewarming and the filtrate is replaced by crystalloid solution to maintain reservoir volume while allowing continuous hemofiltration. After separation from $\mathrm{CPB}, \mathrm{MUF}$ is performed to reverse hemodilution. A similar technique has been termed dilutional ultrafiltration. ${ }^{6}$ In addition to differences in technique, there has been significant variability in the criteria chosen for termination of MUF. A survey of 22 institutes revealed that 10 continued MUF until the circuit contents were completely salvaged, 5 used a time-based criterion, 1 used a hematocrit end point, 1 used a filtrate-volume end point, and 5 used other end points. ${ }^{9}$ The use of varying techniques and end point criteria has made interpretation of published results difficult; nonetheless, the beneficial effects of MUF have been independently reproduced at many institutions.

Concerns have been raised about potential risks and complications of MUF. ${ }^{10,11}$ There was initial concern that MUF would lead to hemodynamic instability by withdrawing blood from the arterial cannula immediately after separation from CPB. Actually, the converse proved true and MUF results in an increase in arterial blood pressure with decreased filling pressures and improved cardiac performance. Multiple studies have demonstrated that concerns over possible complications are primarily theoretical. In their review of MUF at 22 centers, Darling and associates ${ }^{9}$ found no reports of MUF-related morbidity or mortality.

The mechanisms by which MUF produces beneficial effects have not been fully elucidated. Potential mechanisms include reduction of tissue edema, hemoconcentration, and removal of inflammatory mediators. Initially, MUF was hypothesized to improve organ function by simply reducing excess TBW and tissue edema. ${ }^{1,2}$ Studies of the ultrafiltrate, however, demonstrated substantial amounts of inflammatory mediators and vasoactive substances, including inter- leukins 6,8 , and 10 , tumor necrosis factor $\alpha$, and endothelin-1. $6,8,11,12$ One rationale for use of CUF and zero-balance ultrafiltration is to initiate removal of mediators early in the inflammatory cascade and thus decrease the severity of the inflammatory response. ${ }^{6,8}$ Although it is tempting to speculate that removal of these mediators diminishes the inflammatory response to $\mathrm{CPB}$, thus ameliorating some of the adverse sequelae, no study has yet established a definite relationship between removal of inflammatory mediators and improved outcome. The positive benefits of MUF, however, do correlate with the volume of filtrate removed. In a carefully designed study, Daggett and associates ${ }^{13}$ evaluated the effectiveness of no ultrafiltration, CUF, and MUF in preventing tissue edema and organ dysfunction using a neonatal swine model of CPB. MUF was more effective in preventing accumulation of TBW and myocardial edema. MUF also resulted in a significant improvement in left ventricular contractility, assessed by the preload recruitable stroke work index. Interestingly, reinfusion of the filtrate resulted in depressed myocardial function, suggesting the filtrate does contain potentially toxic factors. In a clinical study evaluating the effect of MUF on left ventricular systolic function using load-independent measures of myocardial performance, changes in left ventricular systolic function were shown to correlate positively with the degree of hemoconcentration. The concentration of inflammatory mediators in the filtrate does not differ between CUF and MUF. However, because the volume of filtrate removed is significantly greater with MUF, removal of mediators is correspondingly greater. ${ }^{12}$ Thus, whether the mechanism is reduction in TBW or removal of inflammatory mediators, MUF is more effective then CUF because a greater volume of filtrate can be removed.

In this issue of the Journal, Thompson and colleagues ${ }^{14}$ report results of a study comparing outcomes after CUF and MUF when a standardized volume of fluid is removed. The stated goal of the study was to determine whether "MUF has any intrinsic benefit over CUF aside from the potentially greater volume of fluid removed. ..." The volume of filtrate removed was arbitrarily set at $50 \%$ to $60 \%$ of the "effective fluid balance," defined as the priming volume plus volume added during $\mathrm{CPB}$, less the urine output. Despite the standardization of CPB, CUF patients received a significantly larger priming volume and a larger volume was added during $\mathrm{CPB}$, even though the MUF patients were larger. The reasons for the increased priming volume are unclear; however, it is likely that more volume was added during CPB to maintain the reservoir level during CUF. Because of this additional volume, a significantly greater volume of filtrate was removed during CUF than during MUF (96 vs 69 $\mathrm{mL} / \mathrm{kg} ; P=.01)$. There was no difference in outcome between the 2 groups. The study design suggests a misunderstanding of the rationale for MUF. MUF was introduced 
to allow safe removal of a greater volume of fluid than possible during CUF and thus more effectively prevent accumulation of TBW, not because any special efficacy of ultrafiltration performed after separation from CPB. The composition of the filtrate remains the same whether ultrafiltration is performed during rewarming or a few minutes later after separation from CPB. Indeed, one of the authors of this study stated in a recent editorial that "modified ultrafiltration filters the CPB perfusate in exactly the same way as conventional ultrafiltration, except the filtration process is performed after separation from cardiopulmonary bypass."10 The beneficial effects of MUF compared with CUF are dependent on more aggressive fluid removal. Therefore, a study design that limits the filtrate volume during MUF predetermines the result. Unfortunately, this study adds little useful information to the ongoing debate concerning the optimal use of ultrafiltration during and after CPB.

At The Children's Hospital of Philadelphia, both CUF and MUF are used in neonates and infants during and after $\mathrm{CPB}$, including those undergoing staged reconstruction for hypoplastic left heart syndrome. In an 18-month period, MUF was performed in 467 patients weighing less than 15 $\mathrm{kg}$. The median age was 4 months and the median weight was $5 \mathrm{~kg}$. CUF was used in $87 \%$ of these patients. MUF is continued until the circuit contents have been completely salvaged. The mean volume of ultrafiltrate removed was $129 \pm 60 \mathrm{~mL} / \mathrm{kg}$. The filtrate volume was greater than 100 $\mathrm{mL} / \mathrm{kg}$ in $65 \%$ of patients and greater than $150 \mathrm{~mL} / \mathrm{kg}$ in $30 \%$. The baseline hematocrit value was $38 \% \pm 7 \%$ and decreased to $25 \% \pm 4 \%$ at the end of CPB. MUF resulted in an increase to $40 \% \pm 6 \%$. The mean duration of MUF was $12 \pm 3$ minutes. There were no MUF-related complications.

Multiple studies have demonstrated that ultrafiltration, both during and after CPB in children, is safe and reduces postoperative morbidity, yet many important questions remain unresolved. It is important to recognize that CUF and MUF are not competing, mutually exclusive techniques but rather are complementary techniques with potentially additive positive effects. Filtration during CPB (CUF or zero-balance ultrafiltration) may be used to remove inflammatory mediators and vasoactive substances, whereas MUF is performed after CPB to reverse hemodilution and decrease tissue edema. The optimal use of ultrafiltration in children undergoing repair of congenital heart defects will likely result from a combined technique. The mechanisms by which ultrafiltration results in improved organ function require additional investigation. As the technology and practice of CPB change with decreased use of hemodilution, introduction of smaller circuits, and less use of hypothermia and circulatory arrest, the indications for ultrafiltration are likely to change as well. Further studies are necessary to identify the patients most likely to benefit from ultrafiltration and to define the best protocols for the use of ultrafiltration in these patients.

\section{References}

1. Naik SK, Knight A, Elliott MJ. A successful modification of ultrafiltration for cardiopulmonary bypass in children. Perfusion. 1991;6:4150.

2. Naik SK, Knight A, Elliott MJ. A prospective randomized study of a modified technique of ultrafiltration during pediatric open-heart surgery. Circulation. 1991;84(Suppl):III-422-31.

3. Naik SK, Balaji S, Elliot MJ. Modified ultrafiltration improves hemodynamics after cardiopulmonary bypass in children. [abstract]. $J \mathrm{Am}$ Coll Cardiol. 1993;19:37.

4. Davies MJ, Nguyen K, Gaynor JW, Elliott MJ. Modified ultrafiltration improves left ventricular systolic function in infants after cardiopulmonary bypass. J Thorac Cardiovasc Surg. 1998;115:361-70.

5. Chaturvedi RR, Shore DF, White PA, Scallan MH, Gothard JWW, Redington AN, et al. Modified ultrafiltration improves global left ventricular systolic function after open-heart surgery in infants and children. Eur J Cardiothorac Surg. 1999;15:742-6.

6. Bando K, Turrentine MW, Vijay P, Sharp TG, Sekine Y, Lalone BJ, et al. Effect of modified ultrafiltration in high-risk patients undergoing operations for congenital heart disease. Ann Thorac Surg. 1998;66:821-8.

7. Koutlas TC, Gaynor JW, Nicolson SC, Steven JM, Wernovsky G, Spray TL. Modified ultrafiltration reduces postoperative morbidity after cavopulmonary connection. Ann Thorac Surg. 1997;64:37-43.

8. Journois D, Israel-Biet D, Pouard P, Rolland B, Silvester W, Vouhé P, et al. High-volume, zero-balanced hemofiltration to reduce delayed inflammatory response to cardiopulmonary bypass in children. Anesthesiology. 1996;85:965-76.

9. Darling D, Nanry K, Shearer I, Kaemmer D, Lawson S. Techniques of paediatric modified ultrafiltration: 1996 survey results. Perfusion. 1998;13:93-103.

10. Hanley FL. Commentary: Pulmonary function after modified venovenous ultrafiltration in infants: a prospective, randomized trial. $J$ Thorac Cardiovasc Surg. 2000;119:501-7.

11. Elliott MJ. Modified ultrafiltration and open heart surgery in children. Paediatr Anaesth. 1999;9:1-5.

12. Wang MJ, Chiu IS, Hsu CM, Wang CM, Lin PL, Chang CI, et al. Efficacy of ultrafiltration in removing inflammatory mediators during pediatric cardiac operations. Ann Thorac Surg. 1996;61:651-6.

13. Daggett CW, Lodge AJ, Scarborough JE, Chai PJ, Jaggers J, Ungerleider RM. Modified ultrafiltration versus conventional ultrafiltration: a randomized prospective study in neonatal piglets. $J$ Thorac Cardiovasc Surg. 1998;115:336-42.

14. Thompson LD, McElhinney DB, Findlay P, Miller-Hance W, Chen MJ, Minami M, et al. A prospective randomized study comparing volume-standardized modified and conventional ultrafiltration in pediatric open-heart surgery. J Thorac Cardiovasc Surg. 2001;122:220-8. 